\title{
Exploring key genes and pathways underlying metastasis endometrial cancer based on gene expression microarray.
}

\section{Hui Wang, Xinhui Sun, Shuai Shao, Na Liu*}

Department of Obstetrics and Gynecology, People's Hospital of Linyi City, Shandong Province, PR China

\section{Abstract}

\begin{abstract}
Purpose: To reveal the key genes and pathways involved in the metastasis of endometrial cancer remains.

Methods: Microarray data GSE29436 consisting of 4 progressive endometrial cancer samples and 4 nonprogressive controls were downloaded from Gene Expression Ommibus database. Differentially Expressed Genes (DEGs) were screened out using Limma package in $\mathbf{R}$, followed by hierarchical clustering. Gene Ontology and pathway enrichment analysis were performed for DEGs.

Results: Bioinformatic analysis revealed a total of 65 DEGs between progressive and non-progressive samples. Functional annotation showed that those genes were manly onriched in functions of cell proliferation, MPKA and TGF-beta signaling pathways, whig Nolved with up-regulated genes pleiomorphic adenoma gene $\mathbb{1}$ (PLAG1), Insulin-Like Growth Faetor (IGF2), and down-regulated genes Fibroblast Growth Factor 20 (FGF20) and Thrombospond (THBS4)。

Conclusion: Sixty-five identified genes in progressive colomenall cancer samples were mainly associated with tumor metastasis possibly by enhanging cekproliferation and affecting MAPK and TGF-beta signaling pathways. High expression of P AG1, FGF2 and low expression of FGF20, THBS4, therefore, appear to play an important role in tumorigenesis and progression of endometrial cancer.
\end{abstract}

Keywords: Endometrial cancer, Differentially expessed

Introduction

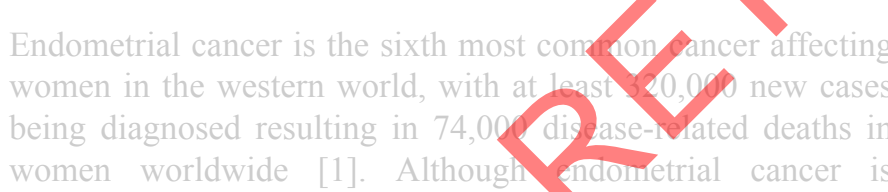

efficiently diagnosed and successfully treated, the treatment of aggressive and progesterone-resistant cancer is difficult However, patients with early stage disease have 5 y survival rates over $80 \%$ but $15-20 \%$ develops metastasis [2].

There are two types of endometrial cancer (types I and II) with different molecular expression profiles and clinical and histopathological behaviors $[3,4]$. Type I endometrial cancer is estrogen-dependent with endometrioid morphology and accounts for $75 \%$ of endometrial cancer [5]. Type II cancer exhibits serious histological alterations and poorly differentiated endometrioid morphology with extra-uterine spread and myometrial invasion. The mechanisms of endometrial cancer metastasis have been partly elucidated, but not fully understood.

In the current study, we aimed to further elucidate the molecular mechanism of endometrial cancer metastasis and provide potential therapeutic target for treating endometrial cancer. Based on the microarray data GSE29436, further analysis was performed by identifying Differentially Expressed
Genes (DEGs) in progressive samples compared with nonprogressive control, as well as functional annotation of DEGs. Finally, functions and involved biological pathway associated with metastasis of endometrial cancer were detected.

Materials and Methods

Data source

Microarray data GSE29436 were downloaded from Gene Expression Omnibus (GEO, http://www.ncbi.nlm.nih.gov/geo/) database based on the platform of Affymetrix Human Genome U133 Plus 2.0 Array. The dataset included 4 progressive endometrial cancer specimen and 4 non-progressive controls.

Data preprocessing and identification of DEGS

The affy package in R [6] was used to process the CEL files by converting the raw probe intensities to probe set expression values. Raw data were background-corrected and normalized with Robust Multi-Array Average (RMA) procedure [7]. Replicate probes for one gene were summarized by mean value as expression value for one gene. To identify critical genes in endometrial cancer metastasis, DEGs were detected using limma package in $\mathrm{R}$ [8] by comparing the expression profile between progressive and non-progressive samples. Batch 